\title{
Ring current influence on auroral electrojet predictions
}

\author{
H. Gleisner and H. Lundstedt \\ Lund Observatory, Box 43, S-22100 Lund, Sweden \\ Received: 25 August 1998 / Revised: 8 March 1999 / Accepted: 17 March 1999
}

\begin{abstract}
Geomagnetic storms and substorms develop under strong control of the solar wind. This is demonstrated by the fact that the geomagnetic activity indices $D s t$ and $A E$ can be predicted from the solar wind alone. A consequence of the strong control by a common source is that substorm and storm indices tend to be highly correlated. However, a part of this correlation is likely to be an effect of internal magnetospheric processes, such as a ring-current modulation of the solar wind $-A E$ relation.

The present work extends previous studies of nonlinear $A E$ predictions from the solar wind. It is examined whether the $A E$ predictions are modulated by the Dst index.This is accomplished by comparing neural network predictions from Dst and the solar wind, with predictions from the solar wind alone. Two conclusions are reached: (1) with an optimal set of solar-wind data available, the $A E$ predictions are not markedly improved by the $D s t$ input, but (2) the $A E$ predictions are improved by $D s t$ if less than, or other than, the optimum solarwind data are available to the net. It appears that the solar wind $-A E$ relation described by an optimized neural net is not significantly modified by the magnetosphere's $D s t$ state. When the solar wind alone is used to predict $A E$, the correlation between predicted and observed $A E$ is 0.86 , while the prediction residual is nearly uncorrelated to Dst. Further, the finding that Dst can partly compensate for missing information on the solar wind, is of potential importance in operational forecasting where gaps in the stream of real time solar-wind data are a common occurrence.
\end{abstract}

Key words. Magnetospheric physics (solar wind magnetosphere interactions; storms and substorms).

Correspondence to: $\mathrm{H}$. Gleisner

e-mail: hansg@astro.lu.se

\section{Introduction}

Variations in the solar wind can be detected at the Earth's surface as small disturbances of the main geomagnetic field. The disturbances are caused by variations in the strength and location of electrical currents flowing in the ionosphere and magnetosphere. These currents are energized by the solar-wind interaction with the magnetosphere and respond dynamically to variations of the solar-wind forcing.

At middle and low latitudes the ring current and the magnetopause currents dominate the geomagnetic records. At higher latitudes, a system of ionospheric electrojet currents and field-aligned currents is more pronounced. The complicated time-varying pattern of geomagnetic disturbances generated by these currents, is transformed into a number of geomagnetic indices that quantify the global level of geomagnetic activity (e.g., Mayaud, 1980; Baumjohann, 1986). Geomagnetic disturbances at low and middle latitudes are monitored by the Dst index at a relatively coarse 1 -h resolution. At higher latitudes, transient disturbances are monitored by the $A L, A U$, and $A E$ indices at a time resolution from one to a few minutes.

The modern definition of a magnetic storm is based on the strength of the ring current, as quantified by the Dst index (Gonzalez et al., 1994). The magnetic substorm is defined from transient geomagnetic disturbances in the auroral zone (Rostoker et al., 1980). Observations show that major storms are always accompanied by intense and frequent substorms, but that substorms can occur in the absence of a magnetic storm. The most intense substorms are usually found within the main phase of storms. In agreement with these observed storm/substorm relations, the $D s t$ and $A E$ indices tend to be correlated (e.g., Davis and Parthasarathy, 1967; Akasofu, 1981; Cade et al., 1995).

The correlation between substorm and storm indices is largely a consequence of the fact that both processes 
are controlled by the solar wind (McPherron, 1997), as demonstrated by the many studies of geomagneticactivity prediction from the solar wind. A part of the correlation is, however, likely to be an effect of internal magnetospheric processes, such as a ring-current modulation of the solar wind $-A E$ relation. In particular, the observed relations between Dst and the location of the maximum electrojet currents (Feldstein, 1992; Feldstein et al., 1997) could play a role.

Predictions of Dst and $A E$ from the solar wind alone demonstrate that magnetic storms and substorms are dynamically controlled by the solar wind. The first of many prediction studies were based on linear techniques: linear cross-correlations between solar-wind parameters and geomagnetic-activity indices (e.g., Arnoldy, 1971; Murayama, 1986) and linear moving-average filters (e.g., Bargatze et al., 1985; Clauer, 1986; McPherron et al., 1988). More recently, nonlinear input-state space reconstructions have been employed (Vassiliadis, 1993; Vassiliadis et al., 1995).

During the last few years, prediction schemes based on artificial neural networks (ANNs) have been developed. The first ANN studies dealt with predictions of the Dst index from hourly averaged solar-wind data (Freeman et al., 1993; Lundstedt and Wintoft, 1994; Wu and Lundstedt, 1996; Gleisner et al., 1996). Correlations between observed and predicted Dst were as high as 0.91 over a large and varied test set covering all phases of the solar-activity cycle (Wu and Lundstedt, 1997). ANNs were found to give better predictions of the Dst index than other techniques.

ANNs were also applied to predictions of the auroral electrojet index $A L$ at 2.5-min resolution (Hernandez et al., 1993), but the results were not conclusive due to a serious clipping problem. Recently, Gleisner and Lundstedt (1997) showed that ANNs can be used to predict the 5-min $A E$ index from solar-wind data, though not with the same high correlation as the hourly Dst index. They also identified an optimal set of solar-wind data ( $n$, $V, B_{y}$, and $B_{z}$ during $100 \mathrm{~min}$ ) for use in $A E$ predictions. Less information on the solar wind than the optimum, led to a decrease of prediction accuracy.

An advantage of the neural network technique is that a very diversified set of input data can be handled simultaneously. Any parameters that contribute information on the solar wind or on the magnetospheric state can be included in the input. The Dst index, either measured or predicted, can be used along with a sequence of solar-wind data as input to $A E$ predictions. If, in fact, the ring current modulates the solar wind- $A E$ relation, we can expect the networks based on both Dst and the solar wind to be superior to networks based on the solar wind alone.

The present paper address two aspects of nonlinear $A E$ predictions. Firstly, we examine whether the $A E$ predictions are improved by $D s t$ when an optimal set of solar-wind data are available. A clear improvement would indicate that the solar wind- $A E$ relation is significantly modified by the magnetosphere's Dst state. Secondly, we examine to what extent Dst can improve predictions when less than the optimum solar-wind data are available. As Dst indirectly contain information on recent solar-wind conditions, it is not unlikely that the Dst index can partly compensate for a loss of solar-wind data. Practical experiences of short-term forecasting based on real-time data (Gleisner and Lundstedt, not yet published) show that loss of information on the solar wind are a common occurrence. One often has to use less than, or other than, the optimum set of solar-wind parameters. Methods to make the networks more tolerant to loss of input information are therefore of potential importance in operational forecasting.

\section{Artificial neural networks}

The following description focuses on the particulars of the ANN models that are used in the present study. A broader view of artificial neural networks can be found in, e.g., Hertz et al. (1991).

\subsection{Network setup}

An artificial neural network is an assembly of interconnected nodes where the strength of the connection between any two nodes is determined by a modifiable weight (Fig. 1). Each node is fed by the sum of the weighted outputs from all the nodes in the previous layer, and pass on the output to all the nodes in the following layer. An additional node, the bias node, is set to 1.0 and connected to all hidden and output nodes in the network. The incoming signal at a node is processed by an activation function, usually a nonlinear, saturating function for a hidden node and a linear function for the output node.

The ANNs used in the present study all have one hidden layer and one output layer. For an input data

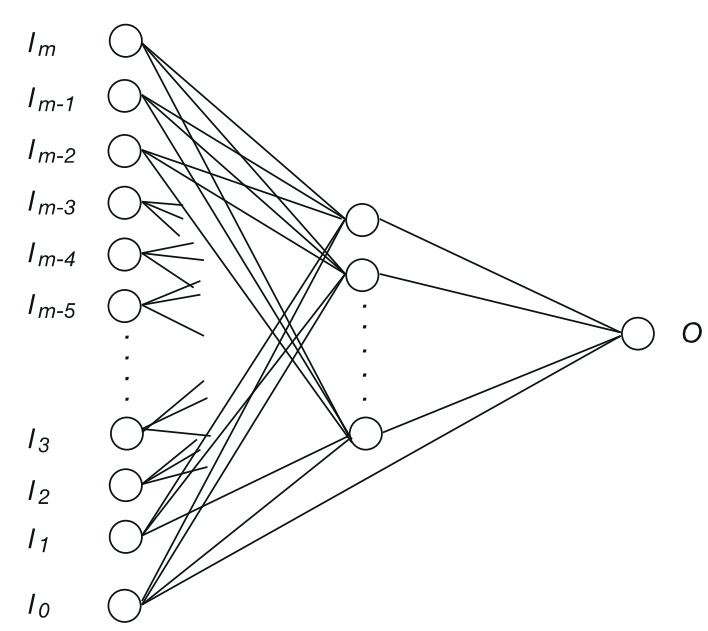

Fig. 1. Network with (left) input nodes and (right) the single output node. The input signal, $\left\{I_{k} ; k=1,2, \ldots, m\right\}$, is propagated to the output, through the hidden nodes where the signal is transformed by nonlinear functions. The input $I_{0}$ is a bias that is set to 1.0 and connected to all hidden and output nodes in the network 
vector, $\left\{I_{k}^{\mu} ; k=1,2, \ldots, m\right\}$, with $m$ components, the network output is given by

$O^{\mu}=g_{O}\left[\sum_{j} W_{j} g_{H}\left(\sum_{k} w_{j k} I_{k}^{\mu}+\theta_{j}\right)+\theta\right]$,

where

$g_{H}(x)=\tanh (x) ; \quad g_{O}(x)=x$.

Each input-output sample $\left\{I_{k}^{\mu}, O^{\mu}\right\}$ is labeled by superscript $\mu$. Index $j$ refers to a hidden layer node, index $k$ refers to an input layer node, and in the output layer there is only a single node. The weight $W_{j}$ thus connects a hidden layer node with an output layer node, while $w_{j k}$ connects input and hidden layer nodes. The terms $\theta_{j}$ and $\theta$ are the weights associated with the bias input $I_{0}$.

\subsection{Network training}

Network training is the process of adjusting the weights until the network produces a response similar to the input-output samples in the training set. The network's ability to produce a correct output is monitored by the cost function

$C(\mathbf{w}) \equiv \frac{1}{2 Q_{\mathrm{trn}}} \sum_{\mu=1}^{Q_{\mathrm{trn}}}\left(O^{\mu}-T^{\mu}\right)^{2}$

where $O^{\mu}$ is the actual output of the network, $T^{\mu}$ is the correct output (or "target"), and $Q_{\operatorname{trn}}$ is the number of samples in the training set.

This nonlinear optimization problem is solved using a modified gradient-descent method referred to as error back-propagation (Rumelhart et al., 1986). The weights are iteratively adjusted according to the gradient-descent rule

$w_{t+1} \leftarrow w_{t}+\Delta w_{t} ; \quad \Delta w_{t}=-\eta\left(\frac{\partial C}{\partial w}\right)_{t}+\alpha \Delta w_{t-1}$,

where $\eta$ and $\alpha$ are constant parameters and $t$ denote the iteration. In each iteration only a subset of the training set is used, and the weights are updated in an approximate gradient direction. This subset consists of $Q_{\text {bat }}$ samples that in each iteration are randomly selected from the set of training data. The three parameters that control the training process have here been assigned the values

$Q_{\text {bat }}=1000$,

$\eta=0.015$

$\alpha=0.90$.

Variations on this basic training procedure are more thoroughly discussed by Hertz et al. (1991).

\subsection{Generalizing with a trained network}

Much of the practical use of neural networks is based on their ability to make sensible generalizations. This ability can be formally defined as the average network performance on a randomly chosen new data sample (Hertz, 1993). The generalization ability can be estimated by the network performance on a test set which contain data that are not used during training.

The training procedure described above optimizes the network's ability to memorize the training data. In order to optimize the generalization ability, the training procedure needs to be constrained. This is done by excluding a small part of the training set from the actual training, and using these data (the validation set) to determine when to stop the iteration. In this way the problem of overfitting is avoided, or at least lessened.

In the present study, a network's generalization ability is quantified by two measures: the correlation coefficient between observed and predicted $A E$,

$r=\frac{\frac{1}{Q_{\mathrm{tst}}} \sum_{\mu=1}^{Q_{\mathrm{tst}}}\left(O^{\mu}-\langle O\rangle\right)\left(A E^{\mu}-\langle A E\rangle\right)}{\sigma_{O} \sigma_{A E}}$,

and the mean-squared error normalized by the variance of the observed $A E$ data,

$V_{\text {rel }}=\frac{\frac{1}{Q_{\mathrm{tst}}} \sum_{\mu=1}^{Q_{\mathrm{tst}}}\left(O^{\mu}-A E^{\mu}\right)^{2}}{\sigma_{A E}^{2}}$.

Here, the averages of the computed output $O$ (i.e., predicted $A E$ ) and the observed $A E$ are denoted $\langle O\rangle$ and $\langle A E\rangle$, respectively, while $\sigma_{O}$ and $\sigma_{A E}$ are the corresponding standard deviations.

\section{Data}

\subsection{Data sources and selection}

The 5-min averaged solar-wind data were obtained from the IMP 8 database at NSSDC. In the present study, we used all intervals of data from a 14-month period (Nov. 1973 to Dec. 1974) that were at least $24 \mathrm{~h}$ long, contained less than $10 \%$ missing data, and contained no data gaps longer than 3 samples (i.e., $15 \mathrm{~min}$ ). This selection gave 40 intervals covering 21600 samples: 32 intervals $(1400 \mathrm{~h})$ were used to train the networks and 8 intervals $(400 \mathrm{~h})$ were used as an independent test of network performance.

The solar-wind data included the proton number density $n$, the wind speed $V$, the three components of the interplanetary magnetic field, $B_{x}, B_{y}$, and $B_{z}$, given in the Geocentric Solar Magnetospheric (GSM) reference system. We also used the southward component of the magnetic field, $B_{s}$, defined as $B_{s}=-B_{z}$ when $B_{z}<0$ and $B_{s}=0$ when $B_{z}>0$.

The $A E$ data were obtained from World Data Center $\mathrm{C} 1$ in England. The original 2.5-min averages were averaged over $5 \mathrm{~min}$ to be consistent with the solar-wind data. The hourly Dst data, uncorrected for the solarwind dynamic pressure, were provided by NSSDC through the OMNIweb database. 


\subsection{Data characteristics}

The period of study (Nov. 1973 to Dec. 1974) was a geomagnetically very active period. The solar wind was largely dominated by long-lasting high-speed streams associated with coronal holes. Within the body of the high-speed streams, periods of large-amplitude Alfvén waves occurred, generating sustained substorm activity (Tsurutani et al., 1995). The period of study also includes one major (Dst $=-204 \mathrm{nT})$ geomagnetic storm, and several moderate $(-100 \mathrm{nT} \leq D s t \leq$ $-50 \mathrm{nT})$ and weak $(-50 \mathrm{nT} \leq D s t \leq-25 \mathrm{nT})$ storms. The occurrences of $A E$ and Dst during the $1800 \mathrm{~h}$ of study are shown in Fig. 2.

The tendency of substorms to become more frequent and intense during magnetic-storm conditions, is demonstrated by the correlation between $A E$ and $D s t$ over the $1800 \mathrm{~h}$ of data: $r=0.58$ based on the 5 -min $A E$ index and $r=0.62$ based on the hourly averaged $A E$.

\section{$4 A E$ prediction studies}

All ANNs in the present study were trained with 16700 samples of the "correct" input-output relation and tested on 4700 input-output samples that were not used during training. One set of networks was trained with the solar-wind quantities used as individual input variables (the solid symbols in Fig. 4). Another set of networks was fed with coupling functions (the solid symbols in Fig. 5). A third set of networks was fed with both $D s t$ and a sequence of solar-wind data (the open symbols in Figs. 4 and 5).
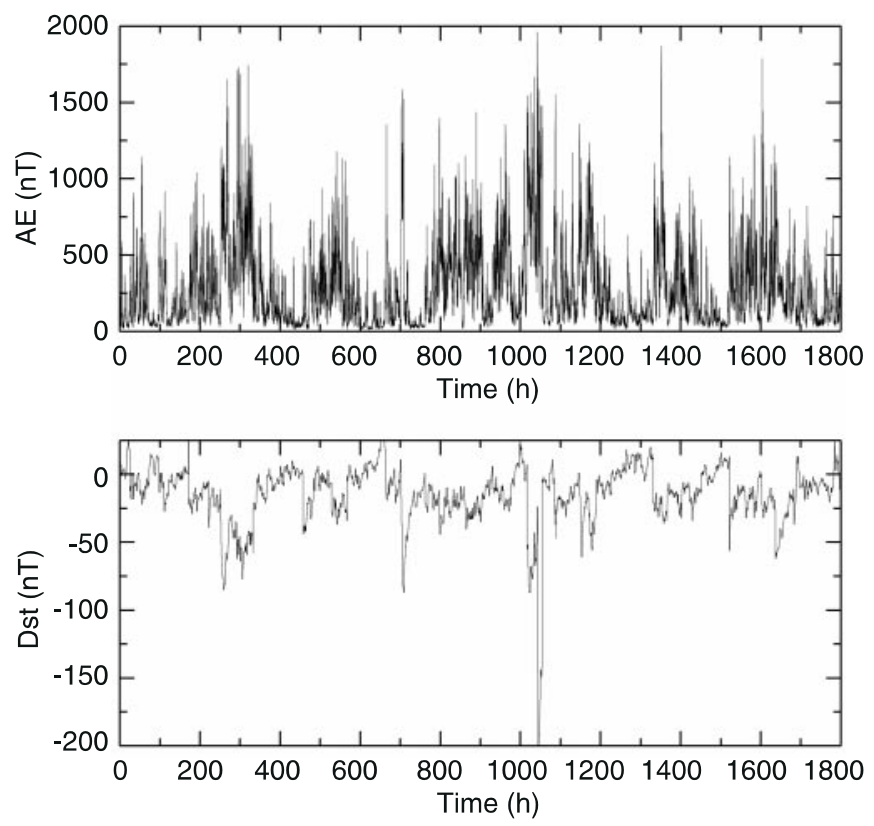

Fig. 2. Occurrences of $A E$ and $D s t$ during the period of study. The data consist of 40 intervals covering $1800 \mathrm{~h}$ selected from the period Nov. 1973 to Dec. 1974

\subsection{Predictions from the solar wind alone}

The basic properties of $A E$ predictions with ANNs that are fed with solar-wind data have been demonstrated by Gleisner and Lundstedt (1997). Figure 3 shows an example of predictions with a network that is fed with 100 min of solar-wind $n, V, B_{y}$, and $B_{z}$. The predicted $A E$ disturbances resemble a smoothed version of the observed disturbances. The accuracy of the predictions depend on the physics encoded into the network: the temporal length of the input sequence and the set of solar-wind variables being fed to the network.

The predictions improve with increasing temporal length of the input sequence for all sets of input variables that are used in this study. Predictions continue to improve up to an input sequence length $T \approx 100 \mathrm{~min}$. For much longer input sequences, the predictions starts to deteriorate as an increased number of weights in the networks makes the overfitting problem worse.

It is evident from Figs. 4 and 5 that there are significant differences between the various combinations of solar-wind parameters. The differences most likely reflect their different abilities to account for the actual mechanisms of energy transfer from the solar wind. The results in Fig. 4 show that it is essential to use all four variables $n, V, B_{y}$, and $B_{z}$. Although $V$ and $B_{z}$ are the most important quantities, the exclusion of $n$ or $B_{y}$ impairs the network performance.

Due to the risk of overfitting, the number of input variables should be as small as possible in order to minimize the number of weights. While the nets must be fed with all relevant information, this information should be given in the form of as few input parameters

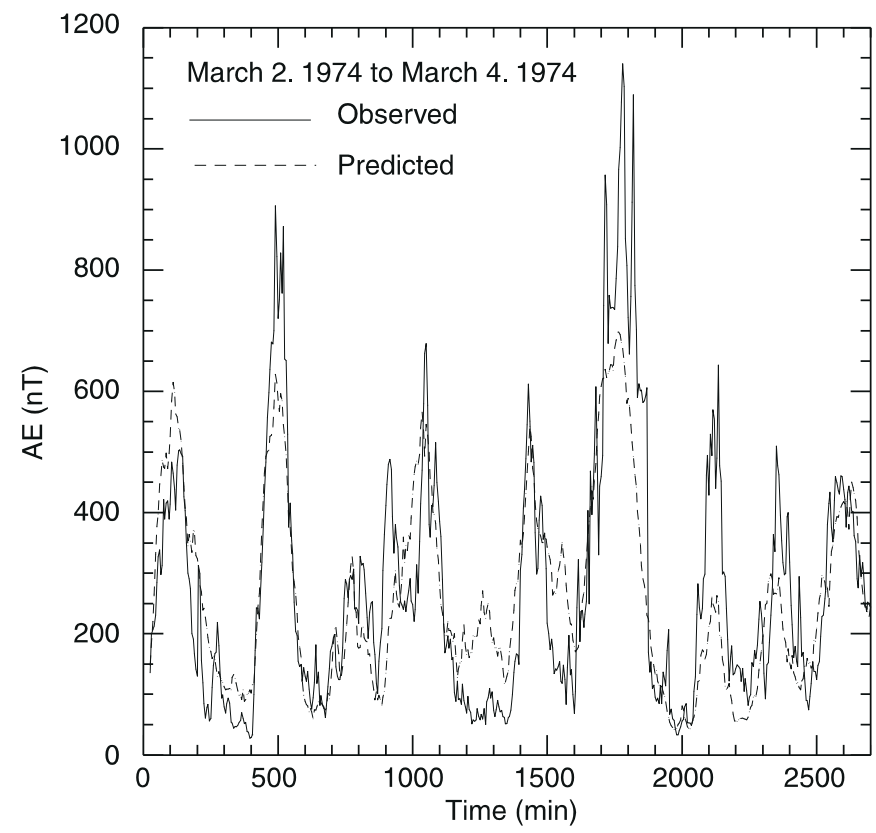

Fig. 3. Observed and predicted $A E$ based on $100 \mathrm{~min}$ of solar-wind parameters $n, V, B_{y}$, and $B_{z}$. This particular interval started on the morning of 2 March 1974 (08.10 UT), and ended two days later on 4 March 1974 (07.15 UT) 


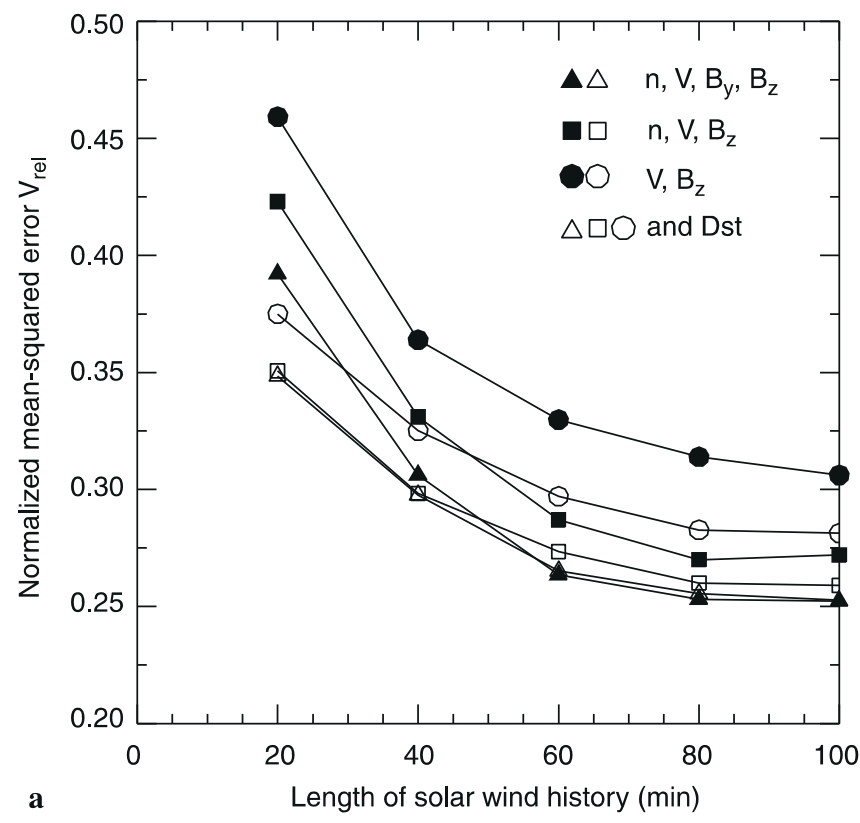

Fig. 4. a Normalized mean-squared error and $\mathbf{b}$ correlation between observed and predicted $A E$, for different sets of solar-wind variables and temporal lengths of the input sequence. The solid symbols mark predictions from the solar wind alone, while the open symbols mark

as possible. The temporal length of the input sequence is determined by the physics of the solar wind-auroral electrojet relation and cannot be reduced in any simple manner. One way of reducing the number of inputs is to combine individual solar-wind variables into coupling functions. This must be done with care, as all coupling functions are not equally relevant as a measure of the coupling between the solar wind and the magnetosphere.

A comparison of networks fed with different coupling functions demonstrate their different abilities to account for the observed $A E$ activity. The function $V B_{s}$ can be interpreted as the rectified dawn-to-dusk component of the solar-wind electric field. It is generally believed to be one of the most important quantities determining the rate of energy transfer from the solar wind to the magnetosphere. The results in Fig. 5 show, however, that the predictions are improved if $V B_{s}$ is properly scaled with velocity $V$ and density $n$. The parameter $V^{2} B_{s}$ scaled with the square root of the solar-wind dynamic pressure, $p \sim n V^{2}$, was the coupling function that gave the most accurate predictions.

In general, the use of individual solar-wind variables is superior to the use of coupling functions. We have still not found a single function of solar-wind parameters that can summarize all relevant information contained in the individual variables. Relevant information on the solar wind is clearly being lost when measured variables are combined into coupling functions.

\subsection{Predictions from the solar wind and Dst}

The predictions described in Sect. 4.1 are based solely on solar-wind data. To each network we now add an

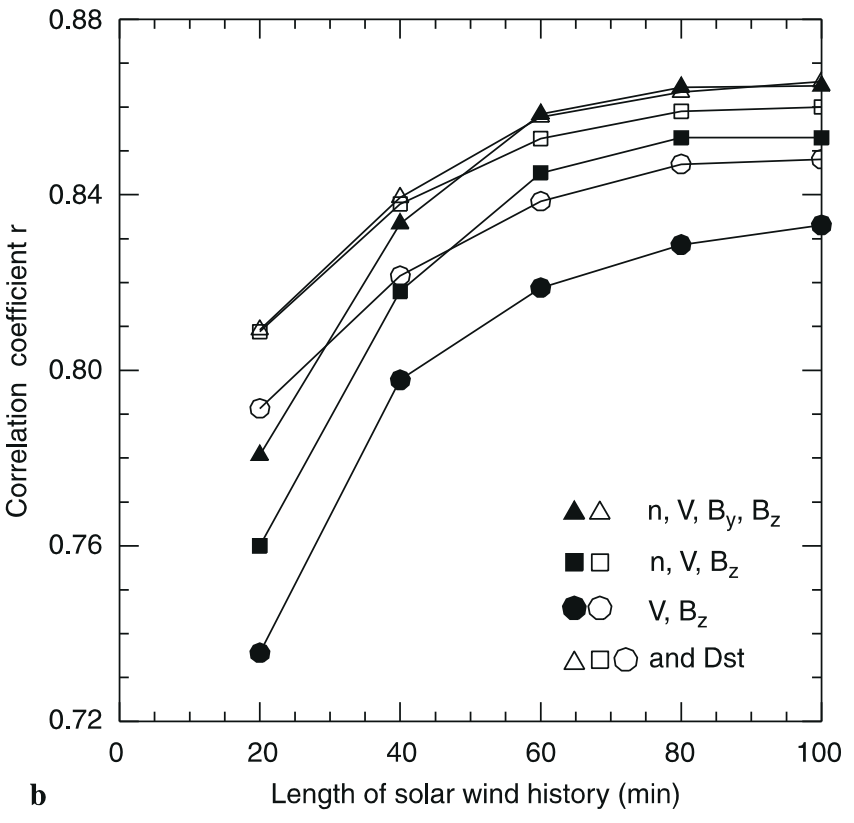

predictions from $D s t$ and the solar wind. The $A E$ predictions are only improved by $D s t$ if less than the optimum solar-wind data are available to the net

additional input node which is fed with the hourly Dst index. The other input nodes are still fed with a sequence of solar-wind data. All networks are trained with data from the same training set as before, and tested on the same test data.

The predictions in this part of the study are thus based on both Dst and the solar wind. Dst contain information on the magnetospheric state, particularly the ring current, but also indirectly on previous solarwind conditions. Improvements of the $A E$ predictions can be the result of the magnetosphere's Dst state modulating the solar wind- $A E$ relation. If the modulation is significant, we can expect a marked improvement of the $A E$ predictions even when the optimum solarwind data are available. However, any improvements of the $A E$ predictions can also be the result of $D s t$ indirectly providing information on the past solar-wind conditions. In this case we expect to find no improvements when the optimum solar-wind data are available, but some improvements when less than the optimum solarwind data are available.

The results of the network runs are shown in Figs. 4 and 5, where the cases with and without the Dst input can be compared (the open and solid symbols, respectively). The influence that $D s t$ has on the $A E$ predictions obviously depends on the amount of solar-wind information that is fed to the network along with the Dst index. The $A E$ predictions are not markedly influenced by $D s t$ when the network has access to at least $100 \mathrm{~min}$ of interplanetary parameters $n, V, B_{y}$, and $B_{z}$. If less solar-wind data are available (Fig. 4), or if coupling functions are used (Fig. 5), the Dst input improves the $A E$ predictions. The more information on the solar wind that is lacking, either due to a too short input sequence 


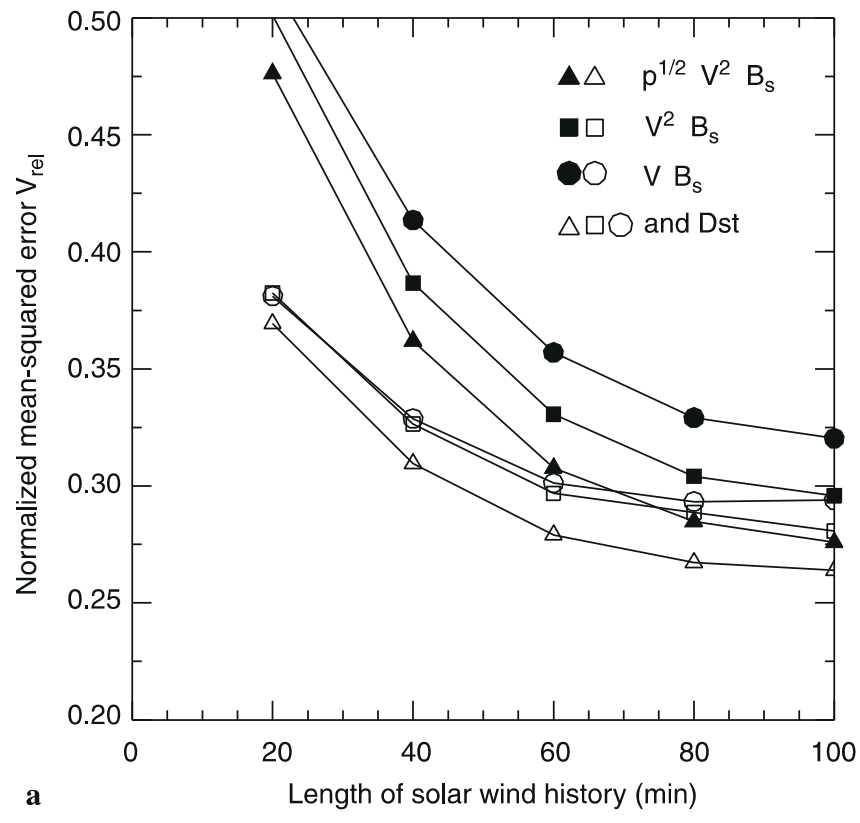

Fig. 5. a Normalized mean-squared error and $\mathbf{b}$ correlation between observed and predicted $A E$, for different coupling functions and temporal lengths of the input sequence. The solid symbols mark predictions from the solar wind alone, while the open symbols mark predictions from $D s t$ and the solar wind. The $A E$ predictions are

or due to relevant solar-wind quantities being left out, the larger the difference is between the cases with and without Dst.

As stated in Sect. 3.2, the linear correlation between $A E$ and Dst is 0.58 over the 21600 training and test samples. When an optimized ANN use the solar wind alone to predict $A E$, the correlation between prediction and observation is 0.86 for data not used during training. The prediction residual is nearly uncorrelated to $D s t: r=0.05$. A large part of the $A E$ variations can thus be explained by a nonlinear mapping from solarwind data without the need to invoke an explicit $D s t-A E$ relation.

We conclude that the ANNs in the present study do not detect a significant $D s t$ influence on the $A E$ predictions when an optimal set of solar-wind data are available. Two identical 100-min sequences of solar-wind data would give nearly the same predicted $A E$ irrespective of the Dst level. This means that the solar wind- $A E$ relation, as described by an optimized ANN, is not significantly modified by the magnetosphere's $D s t$ state.

A second conclusion is that the $A E$ predictions are improved by $D s t$ if less than, or other than, the optimum solar-wind data are available. It appears that missing information on the solar wind is partly compensated by the Dst index. This finding is of potential importance in operational forecasting where gaps in the stream of realtime solar-wind data are a common occurrence.

\section{Conclusions and discussion}

We conclude that the auroral electrojet index $A E$ can be predicted from solar-wind data alone. However, even

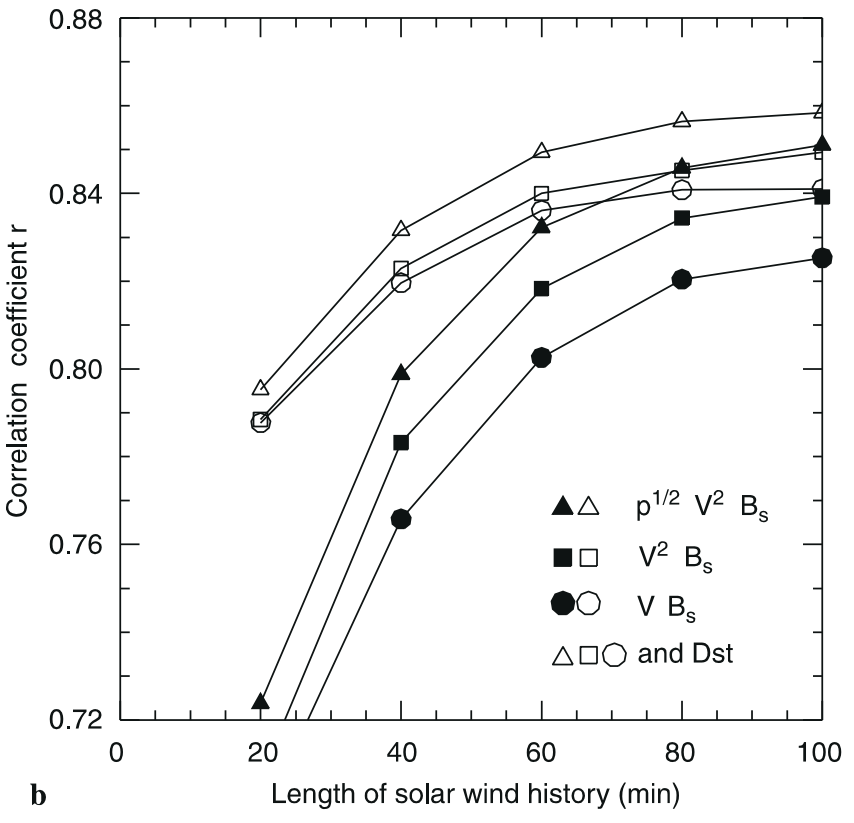

improved by $D s t$ for all the coupling functions tested here. The largest improvements occur when much information on the solarwind conditions are missing. Some information is always lost when measured solar-wind variables are combined into coupling functions

though a nonlinear mapping of solar-wind data can explain a large part of the $A E$ variations, there could still be an independent influence from the ring current acting to modulate the $A E$ index.

From the present study, in which we have compared $A E$ predictions from Dst and the solar wind with predictions from the solar wind alone, two conclusions are reached: (1) with an optimal set of solar-wind data available, the $A E$ predictions are not markedly improved by the $D s t$ input, but (2) the $A E$ predictions are improved by $D s t$ if less than, or other than, the optimum solarwind data are available to the net. It appears that the solar wind- $A E$ relation described by an optimized neural net is not significantly modified by the magnetosphere's Dst state, but that missing information on the solar wind can be partly compensated by the $D s t$ index. When the solar wind alone is used to predict $A E$, the correlation between predicted and observed $A E$ is 0.86 , while the correlation between prediction residual and Dst is very small, $r=0.05$. Thus, we have not been able to detect a significant $D s t$ modulation of the solar wind- $A E$ relation.

The $A E$ index is, by its very definition, not sensitive to the Dst disturbance field generated by the ring current (Mayaud, 1980). Due to the limited latitude coverage of the geomagnetic observatories that are used to estimate $A E$, the $A E$ index depends not only on the strength of the electrojet currents, but also on their location (Kamide and Akasofu, 1983; Akasofu et al., 1983; Baumjohann, 1986). Several studies have shown that the location of the maximum electrojet currents can be observationally related to the strength of the ring current as measured by Dst (Feldstein, 1992; Feldstein et al., 1994, 1997; Popov and Feldstein, 1996; Sumaruk et al., 1989), and also to 
the general level of geomagnetic activity as measured by Kp (Grafe et al., 1983). During time-periods between substorm expansions, a relation between Dst and the latitudes of maximum eastward and westward electrojets is found, whereas the latitude of the maximum westward electrojet during a substorm expansion is not similarly related to Dst (Feldstein et al., 1997). To the extent that the observed Dst-auroral electrojet relations are independent of the solar wind, we would expect the magnetosphere's Dst state to modulate the solar wind$A E$ relation. It appears, however, that the neural nets do not detect such a modulation. A conceivable reason could be that the dependence on the magnetospheric Dst state is relatively weak compared to the solar-wind dependence. It must also be noted that the maximum westward electrojet during substorm expansions, which is not clearly related to the $D s t$ index, is a major contributor to the $A E$ index.

With the availability of real-time solar-wind data from the Sun-Earth libration point L1, short-term forecasting of geomagnetic activity has now become possible. To produce forecasts that are as accurate and reliable as possible, it is important to make use of all data that contain information on the recent solar-wind conditions, but also all data that contain relevant information on the dynamical state of the magnetosphere. In the present work, we have studied the impact of an hourly ring-current index (Dst) that can be accurately predicted, on a high-time resolution auroral-electrojet index $(A E)$. The conclusions show that the Dst index would not markedly improve the forecasts when an optimal set of solar-wind data are available. However, in an operational setting, occasional data gaps are a common occurrence. This is now handled by temporarily using a network with a shorter solar-wind input sequence than the optimum. There are also intervals of time when not all the solar-wind parameters are available. In such less-than-optimal circumstances, the additional information contained in the hourly Dst index can improve the forecasts.

Acknowledgements. National Space Science Data Center and World Data Center C1 for Solar-Terrestrial Physics are gratefully acknowledged for making solar-wind and geomagnetic data available.

Topical Editor K.-H. Glassmeier thanks A. Grafe and A. Klimas for their help in evaluating this paper.

\section{References}

Akasofu, S.-I., Relationships between the $A E$ and $D s t$ indices during geomagnetic storms, J. Geophys. Res., 86, 4820-4822, 1981.

Akasofu, S.-I., B.-H. Ahn, Y. Kamide, and J. H. Allen, A note on the accuracy of the auroral electrojet indices, J. Geophys. Res., 88, 5769-5772, 1983.

Arnoldy, R. L., Signature in the interplanetary medium for substorms, J. Geophys. Res., 76, 5189-5201, 1971.

Bargatze, L. F., D. N. Baker, R. L. McPherron, and E. W. Hones Jr., Magnetospheric impulse response for many levels of geomagnetic activity, J. Geophys. Res., 90, 6387-6394, 1985.

Baumjohann, W., Merits and limitations of the use of geomagnetic indices in solar wind-magnetosphere coupling studies, in Solar
Wind-Magnetosphere Coupling, eds. Y. Kamide and J. A. Slavin, Terra Sci., Tokyo, 3-15, 1986.

Cade III, W. B., J. J. Sojka, and L. Zhu, A correlative comparison of the ring current and auroral electrojets using geomagnetic indices, J. Geophys. Res., 100, 97-105, 1995.

Clauer, C. R., The technique of linear prediction filters applied to studies of solar wind-magnetosphere coupling, in Solar WindMagnetosphere Coupling, eds. Y. Kamide and J. A. Slavin, Terra Sci., Tokyo, 39-57, 1986.

Davis, T. N., and R. Parthasarathy, The relationship between polar magnetic activity $D p$ and growth of the geomagnetic ring current, J. Geophys. Res., 72, 5825, 1967.

Feldstein, Y. I., Modelling of the magnetic field of magnetospheric ring current as a function of interplanetary medium, Space Sci. Rev., 59, 83-165, 1992.

Feldstein, Y. I., A. E. Levitin, S. A. Golyshev, L. A. Dremuhina, U. B. Veshchezerova, T. E. Valchuk, and A. Grafe, Ring current and auroral electrojets in connection with interplanetary medium parameters during magnetic storms, Ann. Geophys., 12, 602$611,1994$.

Feldstein, Y. I., A. Grafe, L. I. Gromova, and V. A. Popov, Auroral electrojets during magnetic storms, J. Geophys. Res., 102, 14223-14235, 1997.

Freeman, J., A. Nagai, P. Reiff, W. Denig, S. Gussenhoven-Shea, M. Heinemann, F. Rich, and M. Hairston, The use of neural networks to predict magnetospheric parameters for input to a magnetospheric forecast model, in Proceedings of the International Workshop on Artificial Intelligence Applications in SolarTerrestrial Physics, eds. J. Joselyn, H. Lundstedt and J. Trolinger, Lund, Sweden, 167-181, 1993.

Gleisner, H., H. Lundstedt, and P. Wintoft, Predicting geomagnetic storms from solar-wind data using time-delay neural networks, Ann. Geophys., 14, 679-686, 1996.

Gleisner, H. and H. Lundstedt, Response of the auroral electrojets to the solar wind modeled with neural network, J. Geophys. Res., 102, 14269-14278, 1997.

Gonzalez, W. D., J. A. Joselyn, Y. Kamide, H. W. Kroehl, G. Rostoker, B. T. Tsurutani, and V. M. Vasyliunas, What is a geomagnetic storm?, J. Geophys. Res., 99, 5771-5792, 1994.

Grafe, A., Electrojet boundaries and electron injection boundaries, J. Geomagn. Geoelectr., 35, 1-15, 1983.

Hernandez, J. V., T. Tajima, and W. Horton, Neural net forecasting for geomagnetic activity, Geophys. Res. Lett., 98, 7673, 1993.

Hertz, J., Generalization in neural networks: theory and practice, in Proceedings of the International Workshop on Artificial Intelligence Applications in Solar-Terrestrial Physics, eds. J. Joselyn, H. Lundstedt and J. Trolinger, Lund, Sweden, 55-64, 1993.

Hertz, J., A. Krogh, and R. Palmer, Introduction to the Theory of Neural Computation, Addison-Wesley, Reading, Mass., 1991.

Kamide, Y., and S.-I. Akasofu, Notes on the auroral electrojet indices, Rev. Geophys., 21, 1647-1656, 1983.

Lundstedt, H., and P. Wintoft, Prediction of geomagnetic storms from solar wind data with the use of a neural network, Ann. Geophys., 12, 19-24, 1994.

Mayaud, P. N., Derivation, Meaning, and Use of Geomagnetic Indices, AGU, Washington, USA, 1980.

McPherron, R. L., The role of substorms in the generation of magnetic storms, in Magnetic Storms, AGU, Washington, USA, 1997.

McPherron, R. L., D. N. Baker, L. F. Bargatze, C. R. Clauer, and R. E. Holzer, IMF control of geomagnetic activity, Adv. Space Res., 8, 71, 1988.

Murayama, T., Coupling functions between the solar wind and the $D_{s t}$ index, in Solar Wind-Magnetosphere Coupling, eds. Y. Kamide and J. A. Slavin, Terra Sci., Tokyo, 119-126, 1986.

Popov, V. A., and Y. I. Feldstein, About a new interpretation of "Harang discontinuity", Geomagn. Aeron., 36, 43-51, 1996.

Rostoker, G., S.-I. Akasofu, J. Foster, R. A. Greenwald, Y. Kamide, K. Kawasaki, A. T. Y. Lui, R. L. McPherron, and C. T. Russell, Magnetospheric substorms: definition and signatures, J. Geophys. Res., 85, 1663, 1980. 
Rumelhart, D. E., G. Hinton, and R. Williams, Learning representations by back-propagating errors, Nature, 323, 533, 1986.

Sumaruk, P. V., Feldstein, Y. I., and B. A. Belov, The dynamics of magnetospheric activity during an intense magnetic storm, Geomagn. Aeron., 29, 110-115, 1989.

Tsurutani, B. T., W. D. Gonzalez, A. L. C. Gonzalez, F. Tang, J. K. Arballo, and M. Okada, Interplanetary origin of geomagnetic activity in the declining phase of the solar cycle, J. Geophys. Res., 100, 21717-21733, 1995.

Vassiliadis, D., The input-state space approach to the prediction of auroral geomagnetic activity from solar wind variables, in Proceedings of the International Workshop on Artificial Intel- ligence Applications in Solar-Terrestrial Physics, eds. J. Joselyn, H. Lundstedt and J. Trolinger, Lund, Sweden, 145-151, 1993.

Vassiliadis, D., A. J. Klimas, D. N. Baker, and D. A. Roberts, A description of the solar wind-magnetosphere coupling based on nonlinear filters, J. Geophys. Res., 100, 3495-3512, 1995.

Wu, J. -G., and H. Lundstedt, Prediction of geomagnetic storms from solar wind data using Elman recurrent neural networks, Geophys. Res. Lett., 23, 319, 1996.

Wu, J. -G., and H. Lundstedt, Geomagnetic storm predictions from solar wind data with the use of dynamic neural networks, J. Geophys. Res., 102, 14255-14268, 1997. 